\title{
A 96-GHz Ortho-Mode Transducer for the Polatron
}

\author{
Goutam Chattopadhyay, Student Member, IEEE, Byron Philhour, John E. Carlstrom, \\ Sarah Church, Andrew Lange, and Jonas Zmuidzinas, Member, IEEE
}

\begin{abstract}
We describe the design, simulation, fabrication, and performance of a 96-GHz ortho-mode transducer (OMT) to be used for the Polatron-a bolometric receiver with polarization capability. The OMT has low loss, good isolation, and moderately broad bandwidth, and its performance closely resembles simulation results.
\end{abstract}

Index Terms-Ortho-mode transducer, Polatron.

\section{INTRODUCTION}

$\mathbf{T}$ HE Polatron is a bolometric receiver with polarization capability, designed for use at the Owens Valley Radio Observatory (OVRO) 5.5-m radio telescope. It will measure the polarization angular power spectrum of the cosmic microwave background (CMB) with high sensitivity and high angular resolution. The polarization of the cosmic microwave background has never been detected [1]. Current models used to describe the intensity variations in the CMB predict a polarization level as high as $10 \%$ of the intensity variations. Many experiments, like the Polatron, are being designed and assembled in the hopes of detecting CMB polarization; such measurements will test our understanding of the origin of $\mathrm{CMB}$ anisotropies and will eventually advance our understanding of the geometry and history of the universe.

The focal plane of the Polatron, shown in Fig. 1, consists of a single-mode scalar feed horn, an ortho-mode transducer to separate orthogonal polarizations of the incoming signal, and cooled spider-web bolometers placed in an ac-bridge for stable readout. The entire instrument will be cryogenically cooled and the bolometers will operate at $250 \mathrm{mK}$. The center frequency of operation of the instrument was chosen at $96 \mathrm{GHz}$ on the basis of the transmission characteristics at the OVRO site, and also to minimize confusion from polarized astrophysical foreground sources, such as galactic synchrotron radiation. The following specification was adopted for the Polatron OMT:

- return loss $\sim 20 \mathrm{~dB}$ or better over $20 \%$ bandwidth for both polarization channels;

- isolation better than $30 \mathrm{~dB}$;

- low insertion loss for both polarizations.

Manuscript received October 8, 1998. This work was supported in part by the Caltech President's Fund under Grant PF414.

G. Chattopadhyay is with the Department of Electrical Engineering, California Institute of Technology, Pasadena, CA 91125 USA.

B. Philhour, S. Church, and A. Lange are with the Department of Physics, West Bridges Laboratory, California Institute of Technology, Pasadena, CA 91125 USA.

J. E. Carlstrom is with the Department of Astronomy and Astrophysics, University of Chicago, Chicago, IL 60637 USA.

J. Zmuidzinas is with the Department of Physics, George Downs Laboratory of Physics, California Institute of Technology, Pasadena, CA 91125 USA.

Publisher Item Identifier S 1051-8207(98)09830-4.

\section{Design, Simulation, AND Fabrication}

There are a few different classes of OMT one could design for the Polatron [2]. The particular choice depends on the performance requirements and fabrication complexity. For the Polatron, we needed a moderately broad-band OMT with good matching and isolation characteristics. From a performance point of view, a "class IIb" OMT described by Bøifot [2] would be ideal. At $100 \mathrm{GHz}$, however, the fabrication of such a device is extremely difficult, because the waveguide dimensions are small and the tolerances are extremely critical. Commercially available OMT's, like Millitech's $W$-band OMT [3], offer $5-10 \%$ bandwidth, which is clearly inadequate for the Polatron. Instead, we decided to design the OMT shown in Fig. 2, which comes under Bøifot's "class Ia" design. The main arm of the OMT has an $F$-band square guide port with a thin septum insert bisecting the common guide. The septum region is partly overlapped by a rectangular waveguide branching, which is located in the center of a perpendicular side wall of the common waveguide with respect to the septum. The broad dimension of the branching waveguide is in line with the longitudinal axis of the common waveguide. The septum acts as a 3-dB power divider for the dominant $\mathrm{TE}_{10}$ mode input signal. At the end of the septum, the signals recombine in the succeeding stages of the waveguide. The orthogonal $\mathrm{TE}_{01}$ mode is evanescent within the septum region; thus, it is reflected back from the septum and is coupled to the orthogonal side arm waveguide. The side arm branching is inductive-iris coupled for the $\mathrm{TE}_{01}$ mode. The inductive iris yields a smaller rectangular aperture size and thus improves matching properties for the $\mathrm{TE}_{10}$ mode. $\mathrm{A}$ capacitive discontinuity in the form of a small step at the bottom wall of the common guide, opposite to the side arm branch, is used to compensate for the influence of the side arm aperture. A standard square to rectangular stepped waveguide transformer is used at the output of the straight $\mathrm{TE}_{10}$ port.

The design parameters for this OMT are the thickness, length, and location of the septum; the thickness and dimensions of the iris; and the width of the capacitive step. Hewlett Packard's high-frequency structure simulator (HFSS) [4] was used to simulate and optimize the design. Ideally, the iris and the septum should be as thin as possible. We chose a 0.001-in thickness for both of them. The septum region must possess a nearly constant short circuit reference plane for the $\mathrm{TE}_{01}$ mode within the desired frequency band. The rejection of the $\mathrm{TE}_{01}$ mode across the septum region should exceed a value of $50 \mathrm{~dB}$ to avoid any impairment by weakly coupled resonances of the waveguide after the septum [5]. Keeping that in mind and also to avoid making the OMT too long, we chose the 


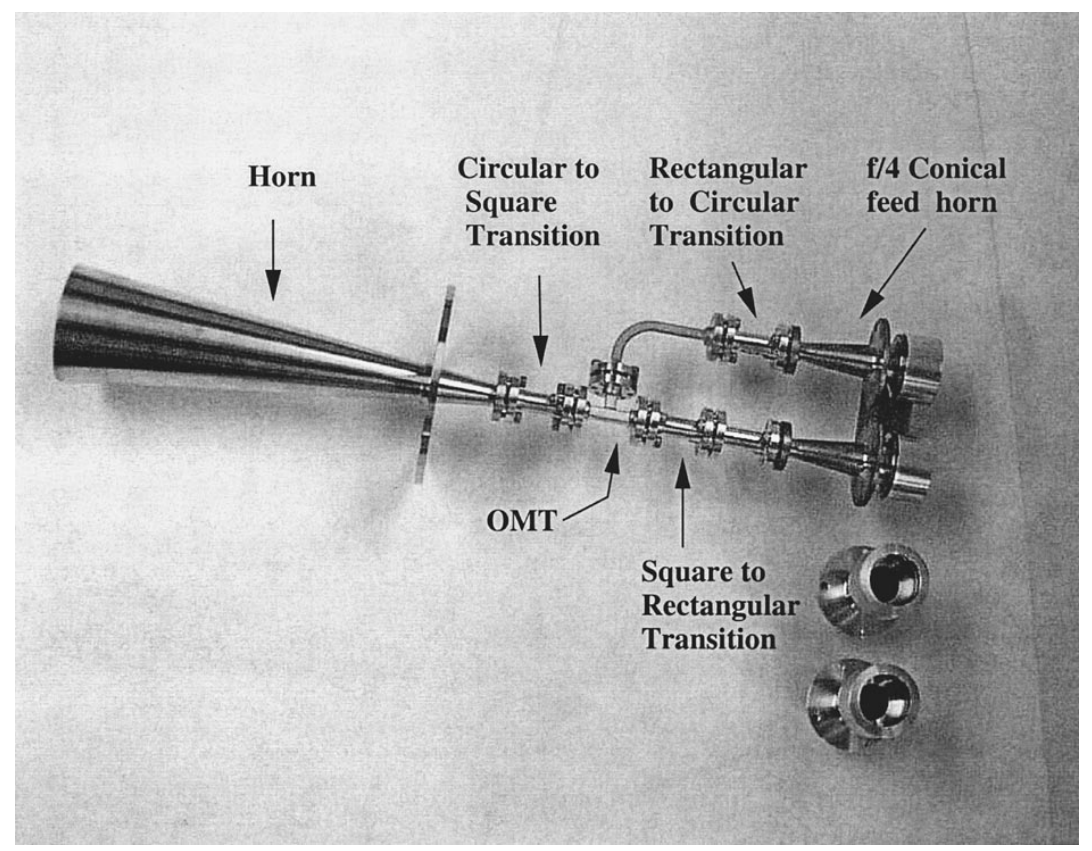

Fig. 1. A picture of the Polatron focal plane. The OMT outputs are fed to $f / 4$ conical feed horns and lenses which form collimated beams. The beams pass through quasi-optical filters and are coupled to bolometers in integrating cavities through another set of horns and lenses.

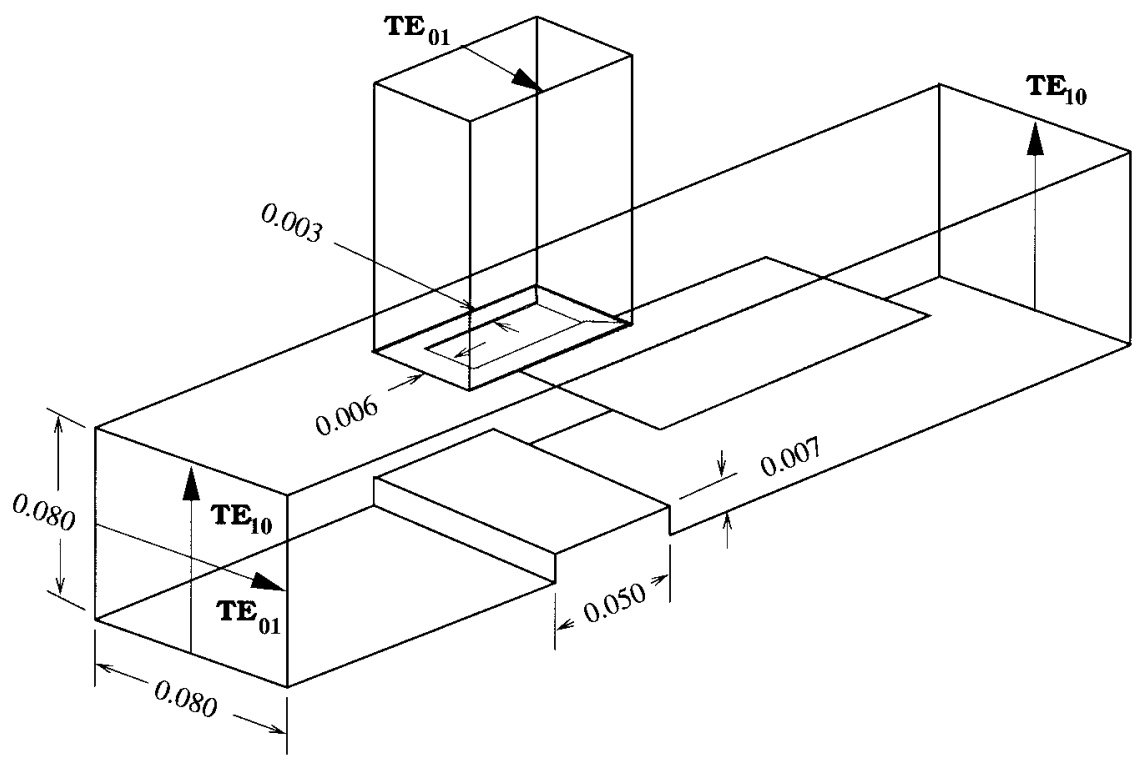

Fig. 2. Detailed sketch of the ortho-mode transducer. The dimensions are in inches.

septum length to be 0.150 in. In our HFSS simulation, all the metals were treated as perfect conductors and under that assumption, the optimum location of the septum was found to be 0.033 in, away from the center of the side arm rectangular guide. The capacitive step and the iris dimensions are shown in Fig. 2. The OMT was fabricated by Hi-Tech Microwaves, Inc. [6] from electroformed copper. There is a groove on the side wall of the guide for inserting the septum, which gives us some flexibility to fine tune the septum location.

\section{RESULTS}

We measured the performance of the OMT at the Jet Propulsion Laboratory [7] with an HP 8510C network analyzer and an $F$-band head. The network analyzer was calibrated with $F$-band waveguide TRL calibration standards. A square to rectangular waveguide transition, which we designed, was used at the input port to mate with the network analyzer head and also to excite the polarization we wanted. The straight port output ( $\mathrm{TE}_{10}$ mode) also had a similar square to rectangular transition. The square to rectangular transitions were not part of the calibration process, and hence, the measurement results have the artifacts of the transitions. To obtain optimum performance, we adjusted the position of the septum; the best performance was obtained with the septum at 0.042 in away from the center of the side arm guide. Fig. 3 shows the simulated and measured return loss performance for 


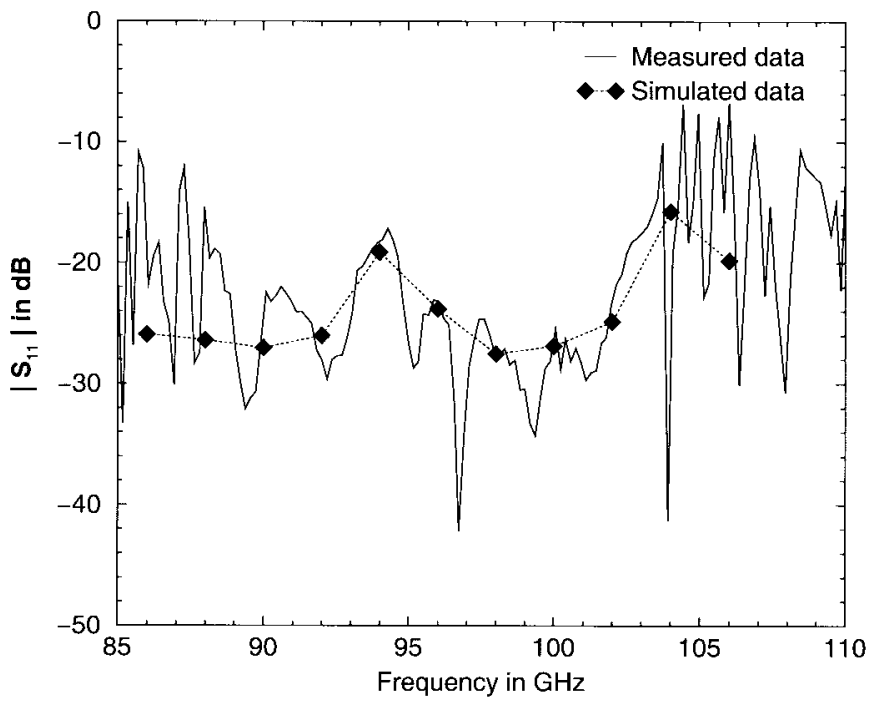

Fig. 3. Return loss performance for TE polarization.

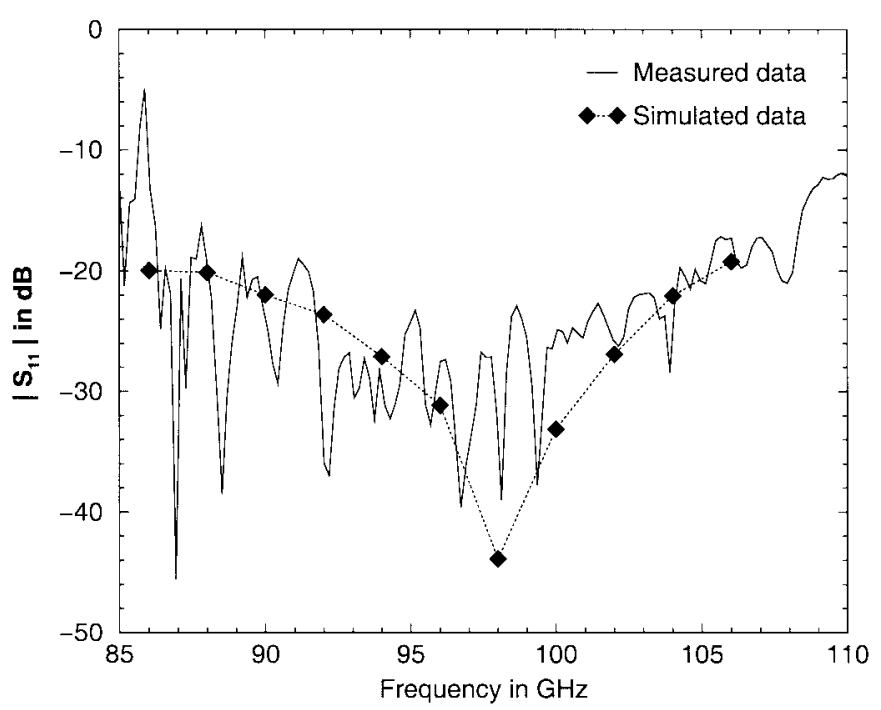

Fig. 4. Return loss performance for TE polarization.

$\mathrm{TE}_{10}$ mode and Fig. 4 shows the same for $\mathrm{TE}_{01}$ mode. Fig. 5 shows straight port isolation, meaning the input polarization was for the side arm port ( $\mathrm{TE}_{01}$ mode) and the output was measured at the straight port. Overall, we found that the measurements closely follow the simulation results. The OMT has low loss for both polarizations; however, since the OMT will be operated at cryogenic temperatures, ohmic loss was not a great concern here.

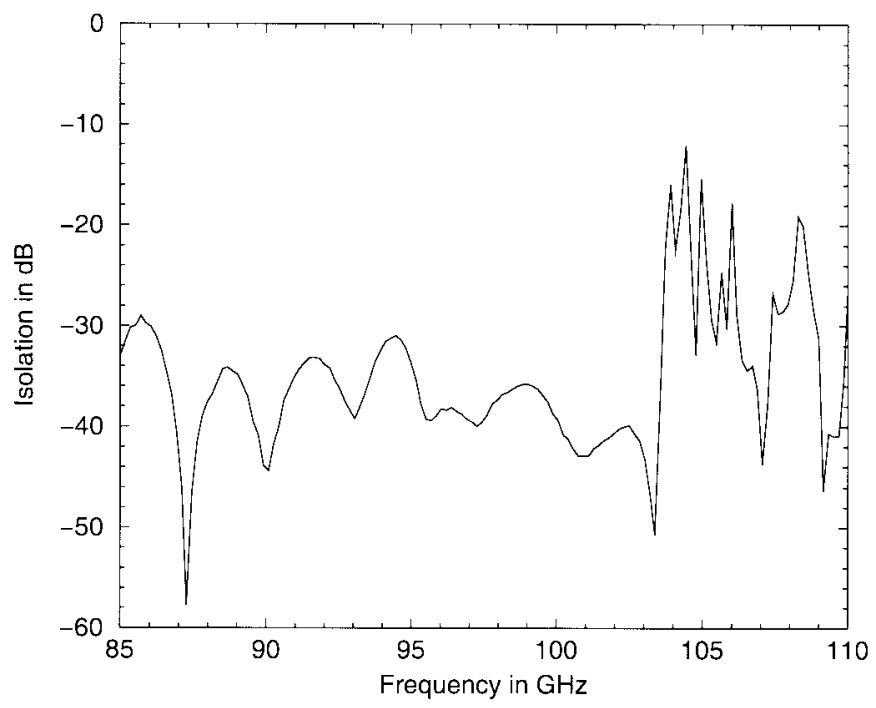

Fig. 5. Isolation performance of the OMT.

\section{CONCLUSION}

The ortho-mode transducer presented here has excellent matching and isolation characteristics over a broad band for both senses of linear polarization. The achieved return loss is less than $-20 \mathrm{~dB}$ over $20 \%$ bandwidth for both polarization channels, and the isolation is better than $30 \mathrm{~dB}$, meeting the requirements for the Polatron instrument.

\section{ACKNOWLEDGMENT}

The authors would like to thank T. Gaier of Jet Propulsion Laboratory, California Institute of Technology, for his assistance with the measurements.

\section{REFERENCES}

[1] W. Hu and M. White, "A CMB polarization primer," New Astronomy, vol. 2, no. 4, pp. 323-344, June 1997.

[2] A. M. Bøifot, "Classification of ortho-mode transducers," Europ. Trans. Telecommunications and Related Technologies, vol. 2, no. 5, pp. 503-510, Sept. 1991.

[3] 1995 Catalog, Millitech Corporation, South Deerfield, MA, ch. 1, p. 45.

[4] High Frequency Structure Simulator (HFSS), version A.04.01, Hewlett Packard Company, Test and Measurement Organization, Palo Alto, CA.

[5] J. Uher, J. Bornemann, and U. Rosenberg, Waveguide Components for Antenna Feed Systems: Theory and CAD. Boston, MA: Artech House, 1993 , ch. 3.8 .

[6] Hi-Tech Microwaves, Inc., Pensacola, FL.

[7] Jet Propulsion Laboratory, California Institute of Technology, Pasadena, CA. 